\title{
Conflictos de valores y conflictos de intereses. La comprensión de la conflictividad en el pensamiento de Julio De Zan
}

\author{
Conflicts of values and conflicts of interest. \\ The understanding of the conflict in the thinking of Julio De Zan
}

Dorando J. Michelini*

\begin{abstract}
Resumen: El artículo expone sintéticamente, en primer término, algunas ideas clave de la teoría ético-discursiva en relación con la resolución de conflictos. Luego se presentan las observaciones críticas que realiza Julio De Zan a la Ética del discurso en el sentido de que los discursos prácticos no serían aptos para resolver los conflictos de valores, los conflictos de intereses y los conflictos político-sociales, como también su propuesta de complementar la Ética del discurso con un aporte hermenéutico y una estrategia de cooperación mutua para la solución de aquellos conflictos que no pueden ser resueltos mediante el discurso argumentativo. Esta estrategia no estaría orientada a alcanzar un consenso entre las partes, sino a lograr un modus vivendi razonable y pacífico. El artículo cierra con una ponderación crítica de las reflexiones de Julio De Zan y con la mención de algunos interrogantes que se plantean a partir de su propuesta de complementación de la Ética del discurso.
\end{abstract}

Palabras clave: conflictividad, Ética del discurso, Julio De Zan, hermenéutica

Abstract: The article summarizes, in the first place, some key ideas of Discourse Ethics theory in relation to conflict resolution. It then presents Julio De Zan's critical observations to Discourse Ethics in the sense that practical discourses would not be suitable for resolving conflicts of values, conflicts of interests and socio-political conflicts, as well as his proposal to complement Discourse Ethics with a hermeneutical

\footnotetext{
* Doctor en Filosofía por la Westfälische-Wilhelms-Universität, Münster. Se ha desempeñado como docente de la Universidad Católica de Santa Fe y de la Universidad Nacional de Río Cuarto, y como Investigador Principal del CONICET. Ha sido becario de las Fundaciones Alexander von Humboldt, DAAD, Friedrich-Ebert y Stipendienwerk Lateinamerika-Deutschland. Posee estudios de postgrado en Filosofía, Ciencias Políticas y Literatura latinoamericana en las Universidades de Frankfurt, Mainz y Münster. Director de la Revista internacional Ética y discurso, de la Red Internacional de Ética del Discurso. Dirección electrónica: dmichelini@arnet.com.ar
} 
contribution and a strategy of mutual cooperation for the solution of those conflicts that cannot be resolved through argumentative discourse. This strategy would not be aimed at reaching a consensus between the parties, but rather at achieving a reasonable and peaceful modus vivendi. The article closes with a critical weighting of Julio De Zan's reflections and with the mention of some questions that arise from his proposal to complement the Discourse Ethics.

Keywords: conflict, Discourse Ethics, Julio De Zan, hermeneutics

\section{Introducción}

La Ética del discurso es una teoría ética que ha tenido repercusión a nivel internacional y también una amplia recepción en América Latina. "Conflicto" y "conflictividad" son dos conceptos que en algunas teorías ético-filosóficas latinoamericanas han sido utilizados como catalizadores para realizar distintas objeciones a la Ética de discurso. Las críticas ponen el foco no sólo en la aplicación histórica del principio moral, sino también en la reflexión éticodiscursiva sobre la fundamentación racional del principio moral. En lo que sigue me referiré a la comprensión de la conflictividad en el pensamiento de Julio De Zan y a las diferencias que muestra su planteo respecto de la propuesta de la Ética del discurso.

Sin desconocer la tematización de los conflictos que tiene lugar en lo que se conoce como la parte "B" de la Ética del discurso apeliana y que refiere

\footnotetext{
${ }^{1}$ Dussel, E., Debate en torno a la Ética del discurso de Apel, México, Siglo XXI, 1994; Dussel, E., "La Ética de la liberación ante la Ética del discurso", Isegoría, 13, 1996, pp. 135-149; Dussel, E., Ética de la liberación en la edad de la globalización y de la exclusión, Madrid, Trotta, 2011; Ibáñez, A., "La Ética del discurso en América Latina”, Espiral, 5(14), 1999, pp. 19-48; Salas Astrain, R., Ética intercultural. Ensayos de una ética discursiva para contextos culturales conflictivos: (re) lecturas del pensamiento latinoamericano, Santiago de Chile, Ediciones de la Universidad Católica Silva Henríquez, 2003; Salas Astrain, R. (coord.), Pensamiento crítico latinoamericano. Conceptos fundamentales, Santiago de Chile, UCSH, 3 tomos, 2005; Michelini, D. J., De Zan, J. y Damiani A. (eds.), Ética, politica y discurso. Contribuciones desde América Latina. Tomo I: Ética del discurso, pragmática universal, pragmática trascendental, lenguaje. Tomo II: Argumentación, discurso, racionalidad, democracia, derecho, religión, Río Cuarto, Ediciones del ICALA, 2015.
} 
a la fundamentación de la aplicación histórica de los principios morales bien fundamentados, De Zan sostiene que hay situaciones -como es el caso de los conflictos de valores y los conflictos de intereses- en las que, más allá de la buena voluntad que tengan los interlocutores para querer resolver sus diferendos de forma discursiva, la naturaleza misma de las diferencias torna difícil, si no imposible, alcanzar entre las partes en litigio un acuerdo discursivo orientado al consenso. Esto se debe, según De Zan, a que el procedimiento del discurso es aplicable a las cuestiones veritativas o a la resolución de los disensos de índole moral, pero no es apto para la solución de conflictos, los cuales no pueden ser dirimidos mediante el discurso argumentativo. "Los discursos prácticos morales son procedimientos cooperativos de búsqueda de la verdad y la justicia, que permiten tratar conflictos de valores, o entre la ética y la moral; los conflictos entre intereses competitivos, las luchas de poder político, y las luchas sociales, etcétera dan lugar a otro tipo de procedimiento que es la negociación y la búsqueda de soluciones políticas". ${ }^{2}$ A diferencia de la estrategia moral de resolución de conflictos -que tiene pretensión de universalidad, tiende a establecer lo que es justo y busca resolver los problemas y conflictos con imparcialidad, poniendo entre paréntesis la conveniencia individual o grupal-, las soluciones pragmáticas y los acuerdos negociados se orientan a resolver problemas parciales y concretos entre algunas de las partes en juego. De Zan está convencido asimismo de que muchos de los conflictos que surgen en el marco de las sociedades modernas de la actualidad -que se caracterizan por el pluralismo de valores (éticos, estéticos, religiosos, etc.) y la búsqueda de una convivencia pacífica entre diferentes formas de vida- no pueden ser resueltos por medio del discurso argumentativo. De Zan entiende la crítica a la capacidad de resolución discursiva de conflictos como un límite de la Ética del discurso y sostiene que es necesario complementar la Ética del discurso con un aporte hermenéutico de comprensión del otro y de las diferencias.

Mi exposición consta de tres partes. En primer lugar, expongo brevemente la teoría ético-discursiva sobre resolución de conflictos (1). Luego presento tres tipos de conflictos que, en opinión de Julio De Zan y a diferencia de lo que propone la Ética del discurso, no pueden ser resueltos por medio del discurso argumentativo, a saber: los conflictos de valores, los conflictos de

${ }^{2}$ De Zan, J., “Identidad y globalización”, en A. Fornari, C. Pérez Zavala, J. Wester (comps.), La razón en tiempos difíciles, Río Cuarto, Ediciones del ICALA, p. 140, nota 11. 
intereses y los conflictos político-sociales. En vista de esta situación, De Zan sostiene que sería necesario complementar la Ética del discurso con un aporte hermenéutico y la elaboración de una estrategia de cooperación mutua para la solución de aquellos diferendos que no son solventables mediante el discurso práctico; esta estrategia no estaría orientada a alcanzar un consenso entre las partes, sino a lograr un modus vivendi razonable y pacífico, lo cual fomentaría la tolerancia y la comprensión de las diferencias (2). Cerraré mi exposición con una ponderación crítica y algunos interrogantes que se plantean a partir de esta propuesta de complementación de la Ética del discurso (3).

\section{Resolución de conflictos en el marco de la Ética del discurso}

La exigencia fundamental de la Ética del discurso expresa que, para llegar a resultados imparciales y justos, todos los problemas y conflictos relevantes deben ser resueltos en los discursos prácticos según el principio del consenso entre los afectados. Antes de abordar la cuestión del rendimiento de los discursos prácticos en relación con la solución de conflictos, quisiera recordar algunas ideas clave sobre los discursos prácticos.

Los discursos prácticos permiten examinar críticamente las pretensiones de validez cuestionadas en vistas a obtener, en el horizonte del consenso racional, ${ }^{3}$ una resolución imparcial de los conflictos; en ellos puede tematizarse todo el espectro de problemas del mundo de la vida, como los problemas de la ética económica y la ética política, las cuestiones bioéticas y los problemas relacionados con la ética medioambiental, etcétera. En los discursos prácticos pueden ponerse asimismo a discusión problemas relacionados con la justicia social, con la justa ponderación de intereses en juego en sociedades complejas y con la implementación de un diálogo intercultural que haga justicia tanto con el reconocimiento del otro como con la resolución justa de conflictos. Entre las principales características de los discursos prácticos pueden mencionarse las siguientes: la inclusión radical de todos los afectados y de todos los intereses y necesidades, la simetría discursiva de los argumentantes y la exclusión de la

\footnotetext{
3 Apel, K.-O., "Falibilismo, teoría consensual de la verdad y fundamentación última" (orig. 1987), en K.-O. Apel, Racionalidad crítica comunicativa, Juan A. Nicolás y Laura Molina-Molina (eds.), Granada, Editorial Comares, 2017, Vol. I, p. 267.
} 
violencia. ${ }^{4}$ Los discursos prácticos son procedimientos falibles y deben permanecer siempre abiertos a revisiones, esto es: a las nuevas consideraciones y propuestas de los afectados. La obligación moral de realizar discursos prácticos en el mundo de la vida no es un deber absoluto. Esto significa que, en principio, nadie debería rechazar la realización concreta de llevar adelante un discurso práctico; sin embargo, puede haber excepciones. Así, por ejemplo, estamos eximidos legítimamente de llevar adelante un discurso práctico con quien nos está amenazando de muerte. Además, en una situación concreta cuando se debe actuar con premura y en solitario-, alguien podría obviar asimismo el discurso práctico real en base a razones que a su entender podrían ser validadas ante la comunidad ilimitada de comunicación y a asumir la responsabilidad que involucra una decisión individual.

Los discursos prácticos se conciben como un procedimiento mediante el cual, en casos de disensos y conflictos, se busca no sólo hallar normas que puedan alcanzar el asentimiento de los interlocutores discursivos, sino también averiguar si los argumentantes están en condiciones de aceptar responsablemente las consecuencias que se desprenden del seguimiento general de lo ponderado como válido en el discurso práctico. ${ }^{5}$ Mediante este procedimiento, la Ética del discurso se abstiene de aportar contenidos morales y orientaciones concretas para la vida, y sólo exige que se examine si el discurso es llevado adelante según las condiciones propias de la comunidad ideal de comunicación.

Tanto Apel como Habermas, si bien con marcadas diferencias, ${ }^{6}$ han dejado en claro que los discursos prácticos son el procedimiento adecuado para resolver no sólo disensos científicos, sino también conflictos prácticos: "El $a$ priori de la argumentación contiene la exigencia de justificar no sólo todas las 'afirmaciones' científicas, sino también todas las exigencias humanas (también las exigencias implícitas de unos hombres a otros, contenidas en las acciones e instituciones)". ${ }^{7}$ Los juicios morales buscan dirimir racionalmente y

\footnotetext{
${ }^{4}$ Habermas, J., Entre naturalismo y religión, Barcelona, Paidós, 2006, p. 88.

${ }^{5}$ Habermas, J., Aclaraciones a la Ética del discurso, Madrid, Trotta, 2000, p. 16.

${ }^{6}$ Habermas, J., Entre naturalismo y religión, pp. 83 y ss.

${ }^{7}$ Apel, K.-O., La transformación de la Filosofía, II: El a priori de la comunidad de comunicación, Madrid, Taurus, 1975, pp. 403, 405.
} 
"solucionar consensualmente"8 los conflictos de acción que surgen en la vida cotidiana. Cuando se trata de resolver litigios entre partes desde el punto de vista moral, la solución pragmática y técnicamente más o menos eficaz entre los participantes que puede aportar la racionalidad estratégico-instrumental no es suficiente; la solución imparcial y justa de conflictos de intereses implica ir más allá de la negociación entre las partes y tomar en consideración las necesidades y los intereses de todos los afectados. En opinión de Habermas, la validez de los resultados de los discursos prácticos no proviene del contexto de acción de los argumentantes, sino del punto de vista moral. ${ }^{9}$ El discurso práctico representa un procedimiento para la resolución de pretensiones de validez cuestionadas. Las materias de regulación discursiva (necesidades, intereses, valores, etcétera) pueden ser imparcialmente resueltas en los discursos prácticos, siempre que ellas sean expresadas como pretensiones de validez. Y a diferencia de lo que sucede en el procedimiento de mediación técnicoinstrumental de intereses, la búsqueda de una solución moralmente relevante de necesidades e intereses se inicia recién cuando se pone en juego un principio de universalización, ${ }^{10}$ según el cual "en las normas válidas, los resultados y los efectos secundarios que se deriven de su seguimiento universal para la satisfacción de los intereses de todos y cada uno tienen que poder ser aceptados por todos sin coacción alguna". ${ }^{11}$ En vista de dicho principio se busca así un consenso entre todos los afectados ${ }^{12}$ y no sólo un entendimiento entre los argumentantes que fácticamente participan en la argumentación. El discurso práctico puede operar además como criterio tanto para la "averiguación discursiva de los intereses de todos los afectados" como para la "diferenciación entre intereses aparentes e intereses verdaderos". ${ }^{13}$

\footnotetext{
${ }^{8}$ Habermas, J., Aclaraciones a la Ética del discurso, pp. 15, 24.

${ }^{9}$ Ibidem, p. 43.

${ }_{10}$ Apel, K.-O., Praktische Philosophie / Ethik: Dialoge 2, Frankfurt am Main, Suhrkamp, 1984, pp. 2, 6, 61 .

${ }^{11}$ Habermas, J., Aclaraciones a la Ética del discurso, p. 16.

12 Apel, K.-O., Diskurs und Verantwortung. Das Problem des Übergangs zur postkonventionellen Moral, Frankfurt am Main, Suhrkamp, 1988, pp. 81 y ss; Apel, K.-O., Praktische Philosophie, pp. 2, 62, 66.

13 Apel, K.-O., Praktische Philosophie, pp. 2, 80.
} 
En síntesis: la Ética del discurso sostiene que una solución imparcial y justa de disensos sobre la ponderación de puntos de vista y conflictos de intereses puede darse solamente como resultado de un discurso práctico racionalmente motivado y orientado al consenso, en el que se tomen en consideración, en el marco de una reciprocidad generalizada, las necesidades e intereses de todos los afectados.

\section{Conflicto de valores, conflictos de intereses y conflictos político- sociales como límites de la Ética del discurso, según Julio De Zan}

En el artículo "Conflictos de valores y de intereses. Dos límites de la ética del discurso", Julio De Zan expone y analiza diferentes tipos de conflictos (conflictos de valores, conflictos de intereses y conflictos político-sociales) que no pueden ser resueltos mediante los discursos prácticos y que, por ende, representarían límites de la Ética del discurso.

\subsection{Conflictos de valores}

Los valores éticos son elementos constitutivos de las culturas y de las diferentes formas de vida particulares que, a diferencia de los principios morales, "no plantean una pretensión de validez universal": en el plano de la eticidad concreta no es posible llevar adelante un discurso en sentido estricto, "porque los enunciados valorativos se apoyan en un trasfondo de creencias y actitudes que no se pueden objetivar mediante enunciados y argumentos racionales". ${ }^{14}$ Además, en sociedades pluralistas, el pedir y exigir razones a quienes tienen una forma de vida diferente, o juzgar las formas de vida diferentes desde los propios estándares de vida, significaría poner en juego "la actitud cargosa e intolerante de quién pretende pedir constantemente explicaciones y justificaciones a los

\footnotetext{
${ }^{14}$ De Zan, J., "Conflictos de valores y de intereses. Dos límites de la Ética del discurso", en R. Maliandi (comp.), Conflictividad. Actas de las Jornadas organizadas por la Academia Nacional de Ciencias de Buenos Aires y la Universidad de Ciencias Empresariales y Sociales, Buenos Aires, UCES, 2010, p. 541, disponible en http://dspace.uces.edu.ar:8180/xmlui/handle/123456789/848.
} 
que piensan y viven de manera diferente". ${ }^{15}$ Esto representa incluso una situación potencialmente conflictiva: "si para poder entendernos e interactuar con los otros en las modernas sociedades pluralistas comenzamos por pedirles las razones de su manera de pensar y de vivir y tenemos que discutir y enjuiciar sus pretensiones de validez, estaríamos bloqueando el entendimiento y generando desde el comienzo una situación potencialmente conflictiva". ${ }^{16}$ En definitiva, el discurso moral no sólo no agotaría el ámbito de lo que puede ser justificado racionalmente, sino que tampoco sería un procedimiento adecuado para resolver determinado tipo de conflictos, como los conflictos de valores.

Los conflictos axiológicos son tan profundos que no pueden ser resueltos mediante el discurso. En el marco de conflictos axiológicos o religiosos, lo que se pone en juego no es negociable, por lo cual las partes no suelen estar dispuestas a ceder en sus posiciones. Es por ello que la tolerancia es a menudo una forma más adecuada de abordar los conflictos interculturales: cuando están en juego valores éticos, valores religiosos o costumbres ancestrales, el diálogo intercultural e interreligioso es más adecuado para comprender las diferencias que los discursos argumentativos, porque aquellos no pretenden alcanzar un consenso, sino que, poniendo en juego la tolerancia mutua, se limitan a la comprensión recíproca.

En los conflictos de valores puede experimentarse así claramente, en opinión de De Zan, una limitación de la aplicación del principio éticodiscursivo, dado que ellos no pueden ser resueltos mediante el discurso argumentativo orientado al consenso. Para superar esta limitación de la Ética del discurso sería necesario complementar el discurso argumentativo con la comprensión bermenéutica, que ayudaría a comprender al otro y las diferencias.

\subsection{Conflictos de intereses}

El discurso argumentativo, al ser un procedimiento orientado a la búsqueda cooperativa de la verdad y la justicia, encontraría igualmente un límite, según De Zan, cuando se trata de resolver conflictos de intereses. Los

\footnotetext{
15 Ibidem, p. 547.

16 Ibidem, p. 546.
} 
conflictos entre intereses competitivos no pueden ser abordados adecuadamente y resueltos apropiadamente por medio del discurso argumentativo orientado al consenso; la solución de conflicto entre intereses necesita de otro tipo de procedimiento, a saber: de la negociación y del aporte de razones pragmáticas.

Para poder ingresar en el discurso argumentativo y tratar cuestiones de validez veritativa y moral, los interlocutores discursivos deben estar interesados -tal como lo ha señalado Habermas- en la búsqueda cooperativa de la verdad y en poner entre paréntesis sus intereses inmediatos de acción; sin embargo, De Zan afirma que estas condiciones no pueden presuponerse en los ámbitos de la competencia y del mercado, porque la conflictividad es un elemento constitutivo en estas esferas de interacción: "la supresión de las relaciones competitivas del mercado, y de las luchas políticas por el poder equivaldría a la eliminación del sistema económico y de la política en sentido moderno, y a su reemplazo mediante la moral y el derecho". ${ }^{17}$

La Ética del discurso, al exigir que las partes renuncien a sus intereses particulares en orden a lograr un resultado imparcial y justo, visualiza la resolución de los conflictos de intereses desde un punto de vista moral; sin embargo, es muy improbable que esta exigencia conduzca al éxito en sociedades donde las empresas, la economía y los partidos políticos difícilmente estén dispuestos a dejar de lado la defensa de sus intereses con el fin de cumplir con el prerrequisito necesario "para ingresar en el terreno de un discurso moral". ${ }^{18} \mathrm{Y}$ en caso de que se lograra poner entre paréntesis los intereses de las partes, la eventual superación del conflicto "no sería el rendimiento del discurso sino de aquella improbable decisión previa de renunciamiento a los propios intereses de las partes"19.

\subsection{Conflictos político-sociales}

\footnotetext{
${ }^{17}$ Ibidem, p. 551.

18 Ibidem.

${ }^{19}$ Ibidem, p. 552.
} 
Con ello quedaría claro, según De Zan, que la concepción del discurso orientado al consenso racional sería inadecuada para la resolución de conflictos políticos y sociales, dado que "los conflictos entre los partidos políticos solamente se pueden superar, en el mejor de los casos, mediante procedimientos discursivos de la racionalidad estratégica, como la negociación de algún tipo de pacto estratégico sobre políticas de Estado y, cuando estas negociaciones fracasan, hay que recurrir al sucedáneo provisorio de la votación". ${ }^{20}$ En política no es posible llevar adelante un discurso orientado al consenso para determinar quién tiene razón, sino que lo que se busca es "cómo evitar la discordia sin lesionar los principios de justicia y atendiendo en lo posible los reclamos razonables de todas las partes". ${ }^{21} \mathrm{El}$ diálogo y la negociación política "operan como ideas regulativas y como alternativas que la democracia antepone al uso de la fuerza en el tratamiento de los conflictos".22

Dado que no parece posible lograr acuerdos sobre lo que es justo si se disiente radicalmente en lo que se considera bueno en un determinado contexto histórico y cultural, la búsqueda de consenso mediante el discurso argumentativo aparece como una ilusión. Más aún, la Ética del discurso incurriría en una cierta inconsistencia, en la medida que "sostiene que la adopción del punto de vista moral de la justicia por parte de los participantes en el discurso práctico, (...) habilita para trascender no solamente las perspectivas orientadas por intereses sino también las basadas en los propios valores culturales". 23

Los conflictos político-sociales marcarían, al igual que los conflictos de valores y los conflictos de intereses, un límite a la Ética del discurso. En los casos de conflictos político-sociales, mediante las negociaciones estratégicas que no están lastradas con la idea de alcanzar un consenso sobre cuestiones de validez, como sucede con el discurso moral- puede obtenerse un saludable acuerdo racional y una convivencia razonable. Las negociaciones políticas, la retórica persuasiva y las razones estrictamente políticas evitan la moralización de

\footnotetext{
${ }^{20}$ Ibidem.

${ }^{21}$ De Zan, J., La vieja y la nueva politica, Buenos Aires, UNSAM Edita, 2013, p. 249.

22 Ibidem, p. 251.

${ }^{23}$ De Zan, J., "Conflictos de valores y de intereses. Dos límites a la Ética del discurso", p. 555.
} 
la política y permiten resolver de forma pragmática los conflictos en el ámbito político-social; por el contrario, "la trasposición directa de la idea del discurso moral, o del discurso filosófico de la verdad al campo de los debates políticos, hace de la Ética del discurso una teoría completamente utópica, incapaz de comprender el tipo de problemas y conflictos de este campo". ${ }^{24}$

La pretensión de universalidad del discurso moral, tal como la comprende Habermas, tiene así, según De Zan, una clara limitación en lo que respecta a la resolución de conflictos en el espacio propiamente político. Que los conflictos políticos y sociales deban tener una solución política significa que el discurso moral no es un procedimiento pertinente para tratar de resolverlos de forma adecuada. Ahora bien, afirmar que el discurso argumentativo orientado al consenso no es un modelo adecuado para la formación racional de la voluntad común y la toma de decisiones en el ámbito público democrático no significa admitir que la alternativa al modelo habermasiano sea la violencia: las luchas por el reconocimiento en el ámbito político y social no implican necesariamente violencia, y las negociaciones y los compromisos pragmáticos de intereses son una alternativa razonable al consenso racional.

En síntesis, De Zan sostiene que el discurso argumentativo es un procedimiento adecuado para tratar problemas morales y cuestiones veritativas, pero es improductivo para la resolución de conflictos de valores, conflictos de intereses y conflictos político-sociales. El procedimiento del discurso argumentativo como medio para la resolución justa de conflictos debería ser complementado con el punto de vista hermenéutico de un diálogo orientado a la comprensión y al entendimiento mutuo de las partes, pero sin la exigencia de alcanzar un consenso. En el caso de conflictos de valores es más adecuado apelar al diálogo comprensivo y la actitud tolerante, que exigir razones; y ante conflictos de intereses y diferendos político-sociales sería más razonable recurrir a las negociaciones estratégicas y a las decisiones pragmáticas, que orientar el comportamiento mutuo de las partes a la búsqueda de consenso. En muchos casos, las negociaciones entre las partes y la mediación estratégica de intereses contrapuestos -más que la búsqueda de consenso- posibilitan acuerdos razonables y una convivencia pacífica. Es por ello que el discurso argumentativo que busca el consenso de todos los afectados debería ser complementado tanto

${ }^{24}$ Ibidem, p. 558. 
con el diálogo orientado a la comprensión hermenéutica y la tolerancia de las diferencias, como con compromisos que no exijan consenso.

\section{Ponderación crítica}

En relación con la problemática de la fundamentación y aplicabilidad de las normas morales en contextos de diversidad cultural, de conflicto de valores y de intereses, y de la problemática de la conflictividad en general, De Zan busca complementar la Ética del discurso sin abandonar los principales logros alcanzados tanto por Apel como por Habermas.

a) Uno de los aportes de Julio De Zan radica en mostrar que el ámbito de la ética es más amplio que el de la moral: la moral, en tanto que procedimiento formal, es indispensable para la resolución justa de conflictos; pero los valores, las tradiciones y las convicciones profundas juegan también un papel relevante en la solución de conflictos. En este sentido, es indudable que la actitud hermenéutica y el acercamiento comprensivo a una cultura extraña es un paso fundamental para el conocimiento del otro y la interacción en la diversidad. Sin embargo, esto no afecta, en mi opinión, la propuesta central de la Ética del discurso que refiere a la resolución justa de conflictos mediante el discurso argumentativo orientado al consenso entre los afectados. $\mathrm{Ni}$ el proceso hermenéutico de comprensión y conocimiento del otro invalida el rendimiento del discurso argumentativo en lo que se refiere al juicio crítico sobre lo que está en juego en cada litigio desde el punto de vista moral, ni la perspectiva moral debe ser concebida como una dimensión enajenante de lo peculiar de cada cultura. Comprensión hermenéutica y ponderación crítica deben ser claramente diferenciadas y, a la vez, valoradas como acciones complementarias.

b) La experiencia muestra que en muchos casos -por ejemplo, ante la necesidad de solucionar conflictos políticos, como asevera De Zan-, la negociación y el acuerdo pragmático pueden ayudar a evitar males mayores. Esta situación podría ilustrarse con la siguiente consideración: muchos conflictos políticos -nacionales e internacionales- son resueltos a menudo por la intervención diplomática y persuasiva de una personalidad científica, política o religiosa, y no a través de una argumentación orientada al consenso. Ante la 
inminencia del conflicto, y sin tiempo o posibilidad de llevar adelante un discurso práctico, es razonable apelar a la negociación y el acuerdo estratégico para la solución del diferendo. Sin embargo, hay que destacar que una solución justa e imparcial de los conflictos -también de los conflictos políticos- no puede provenir legítimamente del poder persuasivo de una personalidad o autoridad, sino del acuerdo libre y racionalmente fundado que alcancen entre sí los afectados. ${ }^{25}$

Los acuerdos y compromisos logrados entre las partes o entre grupos con medios estratégicos pueden ser pragmáticamente útiles, y más o menos eficaces, pero en la mayoría de los casos afectan intereses de terceros y, por ello, no pueden ser considerados como soluciones imparciales. Las negociaciones fácticas entre las partes son relevantes en caso de conflictos sociales, y merecen ser incorporadas como un elemento razonable en la práctica de resolución política de conflictos, pero sólo pueden ser calificadas de justas en la medida que se orienten a aquello que de por sí ellas no pueden lograr, a saber: un entendimiento mutuo -sin exclusiones- entre todos los afectados.

c) La reflexión sobre el ethos ha sido efectivamente poco trabajada por la Ética del discurso: los fundadores de la Ética del discurso se han centrado especialmente en problemas de fundamentación y no de aplicación de la ética. Un aporte interesante en relación con la problemática de la aplicación de las normas en el marco de la Ética del discurso es la distinción que establece Habermas entre las distintas clases de discursos (pragmáticos, éticos y morales). De Zan afirma que habría que complementar la Ética del discurso en el ámbito de la aplicación de las normas morales; por ejemplo, sería necesario incorporar a la Ética del discurso una reflexión hermenéutica de comprensión del otro y de la diversidad, que -más acá de la búsqueda argumentativa de consensoapunte a un entendimiento dialógico con el otro y a lograr una mayor sensibilidad para las diferencias. Así, por ejemplo, en el caso de problemas interculturales y de conflicto de valores, el entendimiento mutuo basado en el

${ }^{25}$ Cfr. Michelini, D. J., "Consenso y conflicto en política”, en D. J. Michelini, R. Hesse, J. Wester (eds.), Ética del discurso. La pragmática trascendental y sus implicancias prácticas, Río Cuarto, Ediciones del ICALA 2009, pp. 77-94; reimpreso en: D. J. Michelini, J. De Zan y A. Damiani (eds.), Ética, política y discurso. Contribuciones desde América Latina, T. II, págs. 134-143. 
diálogo sería no sólo una forma previa, sino eventualmente también más adecuada de solución de conflictos que la que propone la Ética del discurso mediante el discurso práctico.

La reflexión hermenéutica y la razón dialógica constituirían el marco adecuado para tratar todos aquellos asuntos vinculados con el esclarecimiento de los distintos puntos de partida, para la inclusión de los aspectos emocionales que pueden ser relevantes en las relaciones interpersonales e interculturales, para desarrollar una especial sensibilidad respecto de lo particular e individual, para lograr el reconocimiento del otro como requisito para la búsqueda de consenso y, no en último término, para alcanzar un mayor conocimiento histórico, contextual y empírico-pragmático de las partes en diálogo. Se puede coincidir con De Zan en que el esclarecimiento hermenéutico y el entendimiento dialógico son valiosos en sí mismos, porque pueden aportar a un mayor conocimiento mutuo y contribuir también a una apreciación calificada de las diferencias. Sin embargo, me parece que sigue siendo plenamente válida la aseveración ético-discursiva de que una solución "justa”, no meramente estratégica de conflictos de valores, sólo puede alcanzase mediante el discurso práctico. Cuando al interior de una cultura, o en las relaciones interculturales surgen diferendos y controversias axiológicas, lo razonable es explicitar las pretensiones de validez que les son inherentes, de modo que ellas puedan ser analizadas y resueltas de forma imparcial y justa en un discurso práctico. Es por ello que, aun en el marco de los discursos hermenéuticos, las exigencias del discurso argumentativo tienen una fuerza crítica y emancipatoria.

d) Otro asunto importante a discutir es la afirmación de De Zan respecto de que mediante los discursos prácticos pueden resolverse los disensos, pero no los conflictos de intereses. La solución discursiva de los conflictos de intereses requeriría de presupuestos y condiciones tan peculiares, que sería ingenuo pensar que pudieran darse efectivamente. Entre estos presupuestos y condiciones están la disposición de los agentes sociales a poner entre paréntesis los propios intereses y la de entablar un diálogo libre de dominio con los competidores. Sería ingenuo pensar, en opinión de De Zan, que en el ámbito de la economía o de la política las partes en litigio pudieran cumplir con tales condiciones. 
En relación con la aseveración de que es imposible -o al menos muy difícil- una resolución discursiva de los conflictos de intereses, quisiera señalar lo siguiente. Puede haber conflictos de intereses que no sean resolubles discursivamente, porque las partes no están dispuestas a ceder, a poner entre paréntesis sus intereses y a actuar estratégicamente y no como un interlocutor discursivo. Por un lado, se trata de situaciones fácticas, que de hecho se dan a menudo en el mundo de la vida. La Ética del discurso no descarta que puedan darse tales situaciones en el ámbito de las relaciones políticas; más aún, no sería exagerado sostener que en el mundo de la vida la mayoría de los conflictos de intereses se resuelven pragmática o estratégicamente mediante manipulaciones, promesas, amenazas o el uso del poder. Ahora bien, una convivencia justa y solidaria requiere de otros valores que los puramente estratégicoinstrumentales. Para "evitar la discordia" y lograr una convivencia en paz hace falta no sólo la solidaridad y el entendimiento fáctico con el otro, sino también la justicia. Por otro lado, es necesario percatarse de que una sociedad en la que sus ciudadanos no están dispuestos a poner entre paréntesis sus intereses particulares con el fin de debatir sobre su legitimidad y contribuir al bien común, o, en caso de conflicto, a intervenir como interlocutor discursivo para resolver de forma justa y pacífica sus diferendos, tendrá que solventar sus desacuerdos utilizando sólo métodos pragmáticos y estratégicos: con ello se resignaría toda posibilidad de lograr una solución justa de conflictos y de alcanzar una convivencia corresponsablemente solidaria.

e) Dada la pluralidad de valores y formas de vida que tienen lugar en las modernas sociedades democráticas, De Zan interpreta que sería impertinente exigir a los agentes sociales que den cuenta de las mismas con razones que puedan ser comprendidas y analizadas por los demás; esta exigencia se vincularía necesariamente con la intolerancia y provocaría eventualmente situaciones conflictivas.

Al respecto, quisiera destacar que la exigencia que tienen los interlocutores discursivos de dar, recibir y exigir razones en los discursos prácticos no debería ser interpretada como "intolerancia" o como una "actitud cargosa" por parte de los ciudadanos corresponsables que, en el marco de la convivencia democrática, pretenden hallar soluciones justas y pacíficas para los conflictos de intereses y los disensos axiológicos. En el marco de la Ética del discurso, la exigencia de resolución discursiva de las pretensiones de validez, 
lejos de ser una carga insoportable para las partes en litigio, constituye el mejor procedimiento no sólo para abordar conflictos entre normas, sino también para la solución razonable de conflictos de intereses. Es por ello que todos los conflictos de intereses y de valores que puedan ser explicitados como pretensiones de validez pueden ser abordados críticamente y eventualmente resueltos en base a buenas razones en un discurso práctico, esto es: en una argumentación orientada al consenso en que participen todos los afectados.

Más allá de las discusiones a las que puedan dar lugar estas reflexiones, quisiera cerrar mi exposición señalando que Julio De Zan ha sabido no sólo interpretar con agudeza la Ética del discurso, sino también complementar la teoría ético-discursiva con aportes propios. Por lo demás, la obra de Julio De Zan no da lugar a conflicto de intereses ni a conflicto de valores, puesto que ha sido un apasionado buscador de la verdad mediante la discusión pública, un filósofo comprometido con América Latina y uno de esos pensadores sabios que ha hecho siempre de la excelencia un hábito.

Recibido: 02/2019; aceptado: 05/2019 